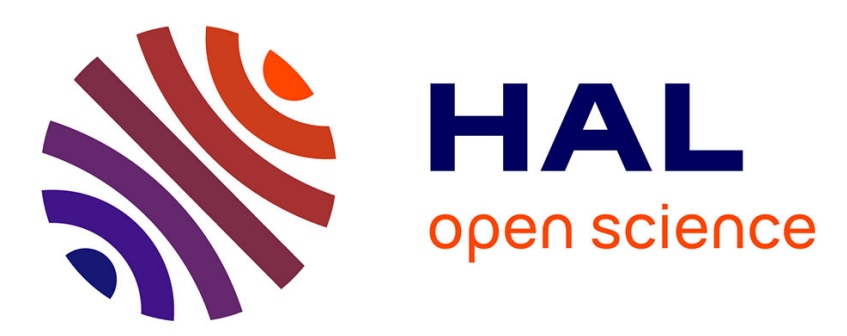

\title{
La politique de transition vers une industrie des TIC sobre en carbone en Chine
}

Gilles Puel, Dai Yue Huang, Chao Liu

\section{To cite this version:}

Gilles Puel, Dai Yue Huang, Chao Liu. La politique de transition vers une industrie des TIC sobre en carbone en Chine. Autrepart - Revue de sciences sociales au Sud, 2014, L'industrialisation au Sud, 2014/1 (69), pp.55-70. 10.3917/autr.069.0055 . hal-01349515

\section{HAL Id: hal-01349515 \\ https://hal.science/hal-01349515}

Submitted on 27 Jul 2016

HAL is a multi-disciplinary open access archive for the deposit and dissemination of scientific research documents, whether they are published or not. The documents may come from teaching and research institutions in France or abroad, or from public or private research centers.
L'archive ouverte pluridisciplinaire HAL, est destinée au dépôt et à la diffusion de documents scientifiques de niveau recherche, publiés ou non, émanant des établissements d'enseignement et de recherche français ou étrangers, des laboratoires publics ou privés. 


\title{
La politique de transition vers une industrie des TIC sobre en carbone en Chine
}

Gilles Puel*,Dai Yue Huang** et Chao Liu***

*MCF HDR en Urbanisme et Aménagement, Université de Toulouse/LEREPS/ETOS, 44, rue Peyrolieres 31000 Toulouse, +33680436607, gilles.puel@univ-tlse2.fr**Doctorant en Économie, Toulouse, Université de Toulouse et de Chongqing, N.14-3, Xinpaifang 1 lu 1 hao, Yubeiqu, 401147 Chongqing China, guyhuang2000@ hotmail.com; ***Professor of School of Power engineering, director of the Thermodynamics Research Institute, Chongqing University, Office.216, School of Power Engineering, Chongqing University, Shapingbaqu, 400030, Chongqing, liuchao@cqu.edu.cn;

Titre court :Chongqing, un modèle en transition

\section{Résumé}

Depuis 1997, le modèle de développement urbain de Chongqing dépend étroitement de la mise en œuvre de la stratégie du Gouvernement central. Après un premier stade basé sur l'industrie lourde, le gouvernement y a encouragé une première transition vers l'industrie des TIC, limitée aux départements de fabrication. Or l'industrie des TIC représente une part importante des problèmes environnementaux de la Chine et du monde. Face à une pression environnementaliste croissante, l'État a décidé d'organiser la transition vers une industrie des TIC à faible impact environnemental et de faire de Chongqing le laboratoire de cette nouvelle stratégie industrielle.Ce papier présente les premiers pas de cette politique de transition en mettant en évidence les leviers et les freins au changement.

Mots-clés : Chine, Chongqing, TIC, développement durable, environnement, modèle de développement.

\section{The Politics of Transition to a Low Carbon IT industry in China}

\begin{abstract}
Since 1997, Chongqing is a model of urban development closely dependent on the implementation of the strategy of the central government. After a first stage based on heavy industry, the government has encouraged a first transition to the IT industry, limited to manufacturing bases. But the IT industry is an important part of the environmental problems of China and the world. Faced with a growing environmentalist pressure, the government has decided to organize the transition to an IT industry with low environmental impact and make Chongqing the laboratory of this new industrial strategy.

This paper presents the first steps of this transition policy by highlighting the drivers and barriers to change.
\end{abstract}

Keywords: China, Chongqing, IT, sustainable development, environment, development model. 


\section{Introduction}

La mise en place d'une gouvernance pour produire un régime global en matière climatique se heurte à de nombreuses difficultés et met en lumière des rapports de force entre pays développés, pour qui une consommation «verte » ou sobre implique une restriction d'accès au marché, et les pays du Sud qui affirment ne devoir réduire leurs gaz à effet de serre (GES) que lorsqu'ils auront atteint un niveau de développement suffisant. L'État chinois a longtemps défendu cette approche, devenant 1' "usine du monde » avec des conséquences désastreuses en matière environnementale suscitant une réprobation internationale mais aussi intérieure.

Face à cettedouble pression protectionnisteet environnementaliste, la Chine, tente de mettre en œuvre une politique de développement durable cohérente avec sa stratégie de développement. Son $11^{\text {ème }}$ plan quinquennal (2006-2010) a donné la priorité aux économies d'énergie, à la réduction de l'intensité énergétique et à l'utilisation de sources d'énergie renouvelables [Lacour et Simon, 2012]. Le 12 ème plan (2011-2015) fixe l'objectif beaucoup plus ambitieux de modifier le modèle de croissance en privilégiant un double rééquilibrage géographique et social interconnecté à un upgrading et un verdissement de ses entreprises. Ainsi l'État chinois a décidé d'organiser la transition vers une industrie des TIC à faible impact environnemental et de faire de Chongqing, métropole du sud-ouest, le modèle territorial de ce nouveau modèle de croissance. La nouvelle zone de LiangJian ${ }^{1}$ en est le laboratoire : " a new area with international influence and demonstration effect for inland opening-up, with modern functions, high-tech industries, gathering headquarters and livable ecology ${ }^{2} \gg$.

Ce papierprésente les premiers pas de cette politique de transition et cherche à mettre en évidence les leviers et les freins à ce changement de modèle.La transition vers une industrie à faible empreinte écologique peut-elle devenir un avantage compétitif pour la Chine ? Eu égard à la spécificité d'un secteur TICmondialisé et largement régulé [Huang et alii, 2012], quels sont les effets du «paradigme » du développement durable dans les politiques publiques aux échelons national et local ? Enfin, quelle est l'originalité du modèle mis en œuvre à Chongqing par rapport aux modèles locaux de développement comme celui du Guandong et est-il transférable?

L'ensemble des données qui ont été utilisées pour cette synthèse ont étérecueillies lors d'une étude empirique ${ }^{3}$, en cours depuis 2008 , portant sur la construction d'un modèle incitatif à l'éco-conception en direction des entreprises du secteur TIC en Chine. Dans ce contexte, les auteurs occupent la position de chercheur embarqué comme expert dans ce processus dirigé par le Ministry for Investments, Industry and Information Technology (MIIT). Cette position au cœur de l'équipe de l'Information Center permet de pallier l'absence de données officielles et d'avoir accès aux documents de travail. Ce groupe rassemble les parties prenantes du secteur TIC et des services concernés de l'État central. Sa mission est double : construire une plate-forme de données fiables et incontestables par les acteurs sur les statistiques en écoinformation des entreprises TIC; proposer, dans un deuxième temps, des scénarios de

\footnotetext{
${ }^{1}$ Il n'existe en Chine que trois zones directement contrôlées par l'État. Les deux autres, à Tianjin (Binhai) et Shanghai (Pudong) existent depuis 1990.

${ }^{2}$ http://english.liangjiang.gov.cn/html/201103/31/content_20416212_2.htm, consulté le 22/01/ 2012.

${ }^{3}$ L'étude s'est focalisée sur 68 grandes entreprises (64 chinoises et 4 étrangères) du secteur TIC, panel construit par le MIIT. Elle est structurée en deux parties : la première relève des éco-informations sur les producteurs, la seconde tente de mesurer leurs motivations et freins dans la transition énergétique. Il s'agit d'une enquête dynamique, lancée en 2009 dont les résultats ont été collectés jusqu'en décembre 2011. Les enquêtes en mandarin ont combiné questionnaires et entretiens approfondis avec des responsables d'entreprises [Huang et alii, 2012].
} 
modèles incitatifs au développement des pratiques de l'éco-conception. L'auteur a ainsi depuis mars 2010 participé aux travaux d'élaboration de la nouvelle réglementation concernant les modalités de perception et d'utilisation du fonds pour l'élimination des déchets électroniques. Chongqing est le terrain des enquêtes et des entretiens auprès des entreprises et autorités administratives.

Après un rappel des principaux enseignements de la littérature sur les rapports entre développement durable et territoire et TIC, ce papier analyse l'importance économiquedes TIC pour la Chine et expose la stratégie de développement du secteur conduite par l'État ;il présente ensuite le modèle de développement de Chongqing, pour montrer qu'il s'insère dans la stratégie plus globale de l'État; enfin, la dernière partie analyse l'originalité du modèle en mettant en exergue points forts et verrous.

\section{Le développement durable, un levier de développement ?}

Il peut sembler paradoxal de vouloir faire en Chine du développement durable un avantage dans la compétitivité mondiale, d'autant que la notion de «développement durable » fait converger des objectifs environnementaux et économiques, mais aussi sociaux, voire de gouvernance [Flipo et alii, 2012] souvent peu conciliables. Surtout en s'appuyant sur une industrie des TIC fortement consommatrice de matières et d'énergie et qui fait souvent peu de cas du recyclage de ses produits : les images d'enfants chinois triant les déchets toxiques des TIC ont fait le tour du monde.

\section{Développement économique, environnement et territoire}

Dans la suite des travaux de Kuznets [1955], Beckerman [1992] établit une relation positive entre le développement économique et le développement durable: "au final, le meilleur moyen - et sans doute le seul - d'atteindre un environnement décent dans la plupart des pays consiste à devenir riches ${ }^{4} \gg$.

À cette conception de la durabilité, dite faible car centrée sur l'économie, s'est opposée une conceptionde la durabilité forte qui envisage l'environnement comme le support de toute activité humaine. Il n'est plus alors considéré comme un simple cadre de vie mais comme un capital naturel limité en amont du cycle de production [Boutaud et alii, 2006].

Ces deux hypothèses s'opposent sur leur approche territoriale du développement durable :

- la première ne se vérifie que si l'on limite spatialement la prise en compte de l'environnement à un même territoire : plus on est riche, plus on porte attention à la qualité de son cadre de vie territorial ;

- la seconde élargit la perspective à l'échelle globale: plus la consommation de ressources est élevée, plus la pression exercée sur l'environnement est potentiellement forte et globale, et liée aux modes de consommation.

Ces oppositions posent la question des transferts territoriaux. Pearce, Markandya et Barbier [1989] évoquent la «durabilité importée ». Un territoire peut améliorer sa durabilité en transférant des externalités négatives (ex : déchets) ou en important (ex : exploitation de ressources «sales ») et ainsi construire sa durabilité aux dépens d'autres. Ce processus s'applique à toutes les échelles : entre local et régional,national ou régional et global. Cette analyse met en avant la durabilité du système global. Nijkamp [1992] introduit la notion de « sacrifices territoriaux » qui pose la question de la légitimité d'une non-durabilité d'un territoire donné au regard du développement durable d'un territoire plus large. Olszak [2012] se réfère aupollution heaven.

\footnotetext{
${ }^{4}$ Cité par Boutaud [2006] p.28.
} 
Le concept de « dette écologique » souligne le transfert de durabilité du Sud vers le Nord[Martinez-Alier, 1993,2002].Ces auteurs affirment que les biens vendus par les pays du Sud, dans le cadre des marchés internationaux, n'intègrent pas l'ensemble des coûts liés à la production et notamment les coûts environnementaux. Guha [1995], s'interrogeant dans le cadre s'un système-monde sur la juste part de nature que chacun peut consommer, oppose les « omnivores » qui consomment beaucoup et rejettent les déchets loin de leurs territoires, aux ecosystem people et aux réfugiés écologiques [Gadgil et Guha, 1995]. Cette approche se décline elle aussi à l'échelle régionale et peut se compléter par une analyse opposant « riches » et «pauvres » [Wallenborn et Dozzi, 2007].

\section{Les TIC, un problème environnemental en soi}

D’une manière générale, le discours dominant affirme que les TIC œuvrent au développement durable bien que les études académiques soient parcellaires, contradictoires et donc peu concluantes [Berkhout et Hertin, 2001] et que l'industrie même des TIC représente une part importante et croissante des problèmes environnementaux du monde. En témoigne la forte régulation (normes, règlementations, labels,...) du secteur initiée par les pays « consommateurs » de TIC et la mauvaise image de la Chine dans ce domaine.

Même si les études sur le sujet demeurent encore rares [Enertech, 2008], Gartner [2008] évalue à $2 \%$ des émissions de GES l'impact des TIC à destination d'un consommateur final à l'échelle de la planète. Cette estimation sous-estime la réalité car elle exclue l'impact des dispositifs numériques embarqués.

La mesure statistique pose problème car la plupart du temps elle est établie sur une base territoriale (nationale ou régionale) qui participe à la construction de représentations. "L'indicateur "intensité carbone" du PIB, par exemple, qui mesure le contenu en carbone d'une unité de valeur ajoutée, avantage les pays fortement tertiarisés, qui n'ont conservé que les bureaux d'études sur leur territoire et consomment des biens qui seraient fabriqués ailleurs » [Flipo et alii, 2012]. L'économie chinoise est ainsi considérée comme particulièrement peu efficace car consommant davantage de ressources pour une même quantité de valeur ajoutée, comparativement à une économie d'un pays développé. C'est oublier que nos modes de vie reposent sur la relocalisation d'activités polluantes au «Sud »et que l'analyse doit aussi se fonder à l'échelle globale sur des données agrégées.Il suffit à la Chine de ralentir l'exploitation des terres rares et de les importer de Malaisie pour améliorer son résultat ! Face à ces difficultés de mesure, Zuindeau [2005] propose de « [...] considérer tour à tour: les importations de ressources naturelles et leurs compensations éventuelles (physiques ou monétaires) ; les transferts d'externalités (exportations de déchets et pollutions transfrontalières) ou de sources d'externalités (industries polluantes); et enfin les conséquences territoriales variables de risques globaux (plus ou moins grande exposition géographique, plus ou moins grande vulnérabilité économique). »

La consommation environnementale des TIC est différenciée territorialement selon des effets de structures liés à la division internationale du travail et aux modes de vie; trois postes peuvent être distingués : fabrication, consommation et recyclage.

Le plus impactant demeure celui de la fabrication, principalement localisée en Asie et surtout en Chine. La part d'un téléphone cellulaire comme d'un ordinateur portable émise lors de sa fabrication est de $80 \%$ contre 20 pour son utilisation. De plus ce secteur est fortement consommateur de matières ${ }^{5}$ [Hagelüken, 2008] : $17 \%$ du palladium, $66 \%$ du tantale, $80 \%$ du ruthenium,... Or les gisements s'épuisent, la demande de terres rares s'accroît [Johnson et alii 2007 ; Bihouix et de Guillebon, 2010] et les coûts d'exploitation menacent d'exploser. La

\footnotetext{
${ }^{5}$ Sans évoquer les toxiques (ex : phtalates ou métaux lourds).
} 
Chine, qui possède l'essentiel des gisements connus, constitue des stocks stratégiques [Flipo et alii, 2012].

Cette situation renouvelle la problématique du recyclage des déchets de la filière qui, quoique marginal pour les GES, tend à devenir un enjeu stratégique. Comment contrôler les flux, le recyclage et les reconquérir sur le secteur informel ?

Pour les pays développés, la part émise lors de l'usage des TIC est prépondérante (Breuil, 2008) et malgré des réglementations de plus en plus contraignantes croît sans cesse à cause de nos modes de vie. Ainsi aujourd'hui la consommation des TIC représente $30 \%$ de la consommation électrique non substituable d'un ménage en France (Enertech, 2008).

\section{L'émergence du développement durable dans le secteur des TIC en Chine}

Dans ce papier, le secteur des TIC $^{6}$ est limité aux activités de fabrication et de services à forte valeur ajoutée, sur la base de la définition de l'OCDE.

\section{Le poids du secteur TIC en Chine}

Son poids dans l'économie nationale s'élève à environ $10 \%$, mais le total en valeur de ses exportations représentait en 2009 plus du tiers des exportations totales chinoises.

Les fabricants locaux (Lenovo, TCL, Huawei,...) se sont développés en conquérant le marché chinois avant dans un second temps de commencer leur expansion mondiale. Ils ont alors dû surmonter un obstacle de taille: le respect des standards environnementaux des pays consommateurs comme les pays européens, les États-Unis, etc. Beaucoup d'efforts ont dû être réalisés en $R \& D$ pour satisfaire ces standards.

Ainsi en 2001, le nombre de brevets déposés en Europe par les producteurs de TIC chinois a dépassé pour la première fois celui déposé aux Etats-Unis ; depuis, la différence est allée croissante. Le marché commun européen, avec sa population de 450 millions d'habitants, est devenu le plus important et le plus dynamique pour les produits TIC chinois. Or, cette région économique reste un territoire où les restrictions commerciales et techniques sont les plus strictes comparativement au reste du monde. En effet, en 2002, l'U.E a annoncé l'application des directives $\mathrm{WEEE}^{7}$ et $\mathrm{RoHS}^{8}$, qui influencent directement l'importation des produits TIC. La chambre commerciale de l'exportation des produits électrique et mécanique chinoise a estimé que le volume financier directement influencé par les deux règlements sur les produits électrique et électronique s'élevait à 32 billions US\$, soit $72 \%$ des exportations du secteur vers l'Europe.

Comme les produits TIC fabriqués en Chine tiennent une place capitale dans le marché mondial, il est devenu stratégique et urgent pour les producteurs-exportateurs de verdir leurs produits et d'orienter leur R\&D selon les règlements européens les plus exigeants.

L'État a décidé d'organiser la transition vers une industrie des TIC à faible impact environnemental

\footnotetext{
${ }^{6}$ L'actuel standard de la classification des secteurs d'activités économiques nationales de la Chine a été publié, en 1994, sous le titre « le standard de classification des secteurs d'activités économiques nationales », et a été renouvelé en mai 2002 (GTB4754-2002), pour une mise en œuvre à partir du $1^{\mathrm{er}}$ octobre 2002.

${ }^{7}$ Waste Electrical and Electronic Equipment, directive européenne de 2002.

${ }^{8}$ Restriction of the use of certain Hazardous Substances in electrical and electronic equipment, directive européenne de 2002.
} 
Pour le gouvernement central, les produits TIC fabriqués en Chinedoivent s'adapter aux standards internationaux, tout en augmentant leur compétitivité dans le marché global [Cong Cao, 2004]; c'est à cette condition que la Chine évoluera de grand pays de fabrication en grand pays de création de produits TIC, et pourra espérer établir un nouvel environnement du marché pour le secteur des TIC à l'échelle mondiale.

L'État a donc entrepris d'organiser la mutation des pratiques de conception et de construire un système d'incitations.La nouvelle loi, Regulations on the recycling and treatment of the Wastes of Electrical and Electronic Equipment (RRTWEEE ) est devenue un projet prioritaire pour le gouvernement, afin de mettre en place une réglementation sur la mise en œuvre de la responsabilité environnementale des producteurs afin de stimuler l'innovation des producteurs pour le marché chinois. Elle ne s'inscrit pas sur une tabula rasa.La plupart des lois, réglementations et standards ont été mis en œuvre au XXI $\mathrm{X}^{\mathrm{e}}$ siècle et couvrent presque tous les éléments du cycle de vie du produit TIC. Elles montrent l'implication récente à améliorer l'efficacité énergétique dans l'industrie.

Mais la grande question demeure de pouvoir contrôler les industriels sur leur respect environnemental pendant le cycle de vie de leurs produits et surtout à la fin du cycle.En effet, notre étudea montré que beaucoup d'entreprises restent en retrait et que la réglementation nationale, peu appliquée, ne motive pas les producteurs pour le marché chinois. Même les entreprises chinoises qui respectent la réglementation européenne pour les produits exportés n'adoptent pas toujours les mêmes pratiques pour le marché chinois [Huang et alii, 2012, 2013].

Aussi le gouvernement expérimente la politique de responsabilité élargie du producteur (REP) sur la fin du cycle de vie de leurs produits par des incitations financières. Le projet introduit trois modèles de responsabilité financière (forfaitaire, collective ou individuelle) selon le degré préalable d'implication du producteur.

Mais ce système peine à s'imposer : résistance des producteurs travaillant pour le marché intérieur, contradiction avec d'autres priorités gouvernementales comme le développement de la consommation intérieure, obstruction des administrations locales qui ont d'autres objectifs que l'environnement et attitude peu favorable des consommateurs.Ces derniers préfèrent les produits les moins chers sans se préoccuper des effets environnementaux. Dans le Guandong, des milliers de petites entreprises, ont, au mépris de tous les règlements, inondé les marchés chinois et mondial (Afrique, Amérique du Sud, reste de l'Asie) de téléphones Shanzhai ${ }^{9}$ [Sun,2012].Quand le gouvernement central a lancé en 2010 un grande campagne de lutte contre ce phénomène (ces entreprises ne paient aucune taxe), le gouvernement local a réagi en modifiant la définition du téléphone Shanzhai, devenu «produit brut d'innovation», préservant ainsi l'essentiel de cet écosystèmeet les emplois locaux.

Ces tensions, amplifiées par un effet de distance en raison de l'immensité de la Chine, entre le gouvernement central et les autorités locales s'inscrivent dans le cadre des questions traditionnelles de la gouvernance multi-niveaux. «Entre les deux sphères (central et local) du pouvoir réel [...] il y a beaucoup d'administration, mais peu d'autorité » [Shue, 1988].Le pouvoir central a toujours tenté de reprendre périodiquement la main sur des gouvernements locaux qui tendent vers l'autonomie : si Shenzhen n'échappe pas à ce modèle [Mercier-Suissa et Ruffier 2012], Chongqing a tissé depuis longtemps des rapports plus étroits avec Pékin et sa politique top down.

\footnotetext{
${ }^{9}$ Fabriqués près de Shenzhen (Huang Qiang Bei), peu chers, le plus souvent de grossières contrefaçons, ils représentent un tiers des téléphones vendus en Chine en 2011, mais aussi 3 millions d'emplois et 10.000 entreprises. Cet écosystème a connu son apogée en 2008, puis une crise en 2010 face au succès des smartphones et à la campagne d'inspection ; aujourd'hui, il est en pleine mutation.
} 


\section{Chongqing cherche un nouveau modèle de développement ${ }^{10}$}

Chongqing ${ }^{11}$ se situe dans le sud-ouest de la Chine, loin des villes côtières mais située à la confluence de la rivière Jialing et du fleuve Yangtze, carrefour stratégique avant la mer. Bénéficiant de cette situation, la ville a connu une première phase de développement quand les nationalistes l'ont choisie comme capitale provisoire. Elle est alors devenue l'une des plus importantes villes pour l'industrie lourde, assurant les bases de son développement industriel. De 1949 à 1997, la ville a perdu son importance économique et politique (Chengdu est devenue la capitale décisionnaire du Sichuan).

\section{Un modèle sous le contrôle du pouvoir central}

En 1997, séparée du Sichuan, elle devient l'une des quatre municipalités administrées directement par le pouvoir central. Ce choix s'explique tant par les avantages de sa situation géographique que par la volonté du pouvoir de mettre en avant sa politique de réduction des inégalités entre les Chines côtière et intérieure. Sa renaissance devait servir de modèle de développement pour l'intérieur de la Chine.

Stratégiquement, l'Ouest doit compenser la baisse de l'attractivité des provinces côtières (en 2012 le salaire minimum est de 1050 yuans contre 1500 à Shenzhen) et éviter les relocalisations d'activités vers les pays à faible coût de main d'œuvre comme le Vietnam. «La mise en valeur de l'Ouest a une histoire de plus de dix ans. Elle a atteint, en partant d'un développement fondamental, un état idéal petit à petit. Le développement économique chinois est caractérisé par le développement de l'Ouest plus rapide que celui du Centre, et celui du Centre plus rapide que celui de l'Est. En tant que pionnier de l'Ouest, le développement de Chongqing fait ressortir cette tendance ${ }^{12} »$. Base manufacturière importante, sa production était destinée principalement à l'armée et jusqu'à une périoderécente, aux classes moyennes chinoises, avec $90 \%$ de ses biens manufacturés vers le marché intérieur.Il n'est donc pas étonnant que son économie soit peu sensible au ralentissement mondial des échanges. «In 2008, when China's growth significantly slowed to only nine percent, Chongqing's GDP grew by $14.3 \%, 5.3$ percentage point higher than that of the national level [Zhiyue, Gang, 2009] ${ }^{13}$.» Cette politique, relancée en 2010 et intégrée au programme national Go West, a subi une inflexion avec le $12^{\mathrm{e}}$ plan. Il s'agit alors non seulement de mettre à niveau mais aussi de changer le mode de développement, de passer d'une économie tirée par les industries lourdes à une économie tirée par les services et l'industrie de la haute technologie, dans un cadre environnemental exemplaire; le «new growth engine for western China » doit tirer parti de ses avantages géographiques pour attirer les entreprises étrangères et faciliter l'intégration économique des régions occidentales de la Chine dans la mondialisation.

Le gouvernement central investit des capitaux considérables pour améliorer les infrastructures de transport. Ainsi une chaîne logistique internationale, la «nouvelle route de la soie », relie

\footnotetext{
${ }^{10}$ La plupart des données sont issues de la thèse de Huang [2013].

${ }^{11}$ En 2012 l'agglomération compterait 10 millions d'habitants dont une moitié de population flottante. Chongqing, plus grande ville du monde, est un mythe construit de toute pièce par les médias qui ont confondu population urbaine et population de la région administrative, appelée Municipalité.

${ }^{12}$ Huang Qifan, maire de la municipalité de Chongqing, 8/11/2012.

${ }^{13}$ En 2008, lorsque la croissance de la Chine a sensiblement ralenti à seulement $9 \%$, la croissance du PIB de Chongqing s'est élevée à $14,3 \%$, soit 5,3 point de mieux (traduction de l'auteur).
} 
l'Europe et l'agglomération en 13 à 16 jours (le chemin de fer international ChongqingXinjiang-Europe). Une «route de la soie électronique» avec un «port international d'information » et quatre aéroports sont prévus pour compléter le système. Le programme « One-Hour Economic Circle » structure les transports et l'organisation spatiale de l'industrie à l'échelle de l'agglomération (2007-2020). En mai 2012, 200 des 500 plus grandes entreprises mondiales s'étaient installées à Chongqing, et parmi elles, la plupart des leaders du secteur TIC (voir tableau 1).

Mais il ne faut pas réduire ce modèle à une simple réplique, plus à l'Ouest, du modèle de développement du Shandong. L'État central ambitionne de faire de Chongqing l'interface entre le monde et son potentiel marché intérieur «the vast markets in central and western China set the stage for the Liangjiang New Area to spearhead growth in these regions ».

\section{La montée en gamme dans l'industrie des TIC}

Le développement (2010) de la nouvelle zone de Liangjiang (1200 km2) est aujourd'hui l'élément clé de cette politique d'intégration dans le marché mondial. Résultats et objectifs annoncés sont impressionnants. Au premier semestre 2012, le PIB de la zone a atteint 72,5 milliards de yuans, $(18,4 \%$ de hausse sur un an). La valeur du commerce extérieur a totalisé 9,1 milliards de dollars (2,6 fois plus que l'année précédente).

Partie intégrante de Liangjiang, le parc industriel high-Tech de Chongqing Shuitu accueille une partie des entreprises du secteur TIC. En 2015, Chongqing vise à devenir la plus grande base de fabrication d'ordinateurs portablesd'Asie (150 millions de machine/an soit le tiers du marché mondial, 1 billion de yuans).L'industrie de l'électroménager (Haier, Gree et Midea) doit se déplacer en Chine occidentale.Les industries de l'électronique numérique complètent les activités de fabrication (ex : 30 millions d'imprimantes en 2015).

La politique de montée en gamme concerne l'Internet mobile, la communication sans fil et l'Internet des objets. Chongqing doit devenir la plate-forme nationale pour la certification et le test des produits de communication, d'améliorer les chaînes de l'industrie en fonction des composants et des matériaux électro professionnels. L'objectif en 2015 est de produire entre autres, 2 millions de serveurs, 50 millions de téléphones IP et 100 millions de modules sans fil par an.

\begin{tabular}{|l|l|l|}
\hline 1998 & Ericsson & $\begin{array}{l}\text { Équipements de communication à micro- } \\
\text { ondes }\end{array}$ \\
\hline 2006 & Oracle & Filiale \\
\hline 2007 & NTT DATA & Xiyong Microelectronic Industry Park \\
\hline 2009 & Deloitte & Filiale \\
\hline 2009 & Foxconn & Ordinateurs portables \\
\hline 2009 & HP & $\begin{array}{l}\text { Ordinateurs portables. Relocalisation } \\
\text { Singapour. }\end{array}$ \\
\hline 2009 & APL & Centre de traitement \\
\hline 2009 & Inventec & Ordinateurs portables \\
\hline 2010 & Quanta & Ordinateurs portables \\
\hline 2010 & CISCO & Équipement de communication \\
\hline 2010 & ACER & Base de production et second siège social \\
\hline
\end{tabular}




\begin{tabular}{|l|l|l|}
\hline 2011 & Pacnets & Cloud computing \\
\hline
\end{tabular}

Tableau 1. Les principales entreprises étrangères du secteur TIC installés à Chongqing (source : auteur)

Enfin, Chongqing veut devenir le «Cloud Computing Data Center Offshore» le plus grand en Asie. Une zone spéciale couvre un terrain d'environ 10 kilomètres carrés dans la zone de New Liangjiang. 100 milliards de dollars sont attendus en 2015. L'industrie des logiciels et des services d'externalisation associésdevraitrapporter 180 milliards de yuans (10\% du total des recettes nationales de ces industries).

\section{Freins et leviers}

Si l'on analyse ce modèle de développement à l'aune de chacundes piliers du développement durable le bilan est mitigé.

Sur le plan environnemental, l'image de Chongqinq est désastreuse. Son passé industriel lui a attribué le titre de «capitale des pluies acides »: depuis 1949, les arbres de la ville ont dû être replantés quatre fois. La situation a été un temps aggravéepar les poussières des chantiers du processus de démolition-reconstruction que suivent les agglomérations.

Aujourd'hui, les autorités locales sont conscientes qu'il est nécessaire de rendre Chongqing attractive,car elles espèrent faire venir de la main d'œuvre qualifiée ou empêcher celle qui est formée sur place de fuir vers les grandes villes côtières ou l'étranger. D'immenses progrèsont été réalisés ou sont programmés comme la nouvelle zone de Liangjiang, composée pour moitié d'espaces verts et d'activités dites propres.

Mais la mise en œuvre de la transition a consisté premièrement (comme dans les autres provinces chinoises développées) à cacher la poussière sous le tapis et à relocaliser les activités les plus polluantes (cimenteries, aciéries, etc.) dans les villes environnantes avec la bénédiction d'autorités locales qui trouvent ainsi un moyen facile de s'enrichir, voire à imposer aux industriels de l'acier le respect des normes de pollution dont l'application dépend de la volonté politique. Il ne faut donc pas s'étonner si à l'échelle de la Chine les cartes des relevés de la pollution de l'air montrent une situation qui empire.

Deuxièmement, des mesures coercitives ont banni le chauffage au charbon, ou inciter les taxis à modifier leur motorisation, etc.Le résultat de cette politique est visible : le soleil réapparaît dans le ciel et il n'est plus nécessaire de laver son parapluie en rentrant chez soi comme l'avaient appris les écoliers à cause des pluies acides.

Troisièmement, la prise de conscience des habitants progresse et ils exercent pression et surveillance via les réseaux sociaux ou des associations. Désormais, Les autorités les informations sur la qualité de l'air sont accessibles sur un terminal mobile ou Internet ${ }^{14}$.

Enfin, quatrièmement, il s'agit de faire venir des industries peu émettrices. La tâche n'en est qu'à ses débuts et reste immense. «If Chongqing can succeed in transforming into a green, low-carbon city, there is no doubt that the rest of Chinese cities will be able to make this switch. ${ }^{15} \gg$ Et la partie est loin d'être gagnée. La plupart des industriels locaux que nous avons rencontrés, n'entrevoient souvent que la dimension économique du développement durable et ne sont sensibles qu'aux investissements dans l'environnement qui génèrent des économies d'énergie à court terme. Aussi pour aller plus loin, le gouvernement, comme avec la REP pour l'industrie des TIC, envisage de d'introduire un système de plafonnement et d'échange de droits à polluer. Les usines émettrices au-delà d'une limite fixée devront acheter des quotas

\footnotetext{
${ }^{14}$ http: //www.aqicn.info/? city=Chongqing, consulté le 25/01/2013.

${ }^{15} \mathrm{Li}$ Yong (Chongqing Academy of Social Sciences). Le processus concerne de plus en plus de métropoles [Li et alii., 2012].
} 
aux plus efficaces, ou parrainer des plantations ou bien payer les agriculteurs qui transforment les déchets en énergie.

Les discours officiels se focalisentpourtant moins sur la dimension environnementale que sur les critères exclusivement économiques (croissance du PIB, taux de capital national investi, nombre d'entreprises du «top $500 »$ (Fortune 500 Global) installés, progrès du taux d'urbanisation, etc.) quimettent en avant le succès de leurstratégie industrielle. Les autorités se félicitent de chaque contrat signé avec une transnationale du top 500. Fin 2012, plus de 200 s'étaient installées à Chongqing. L'arrivée de ces investisseurs ne doit pas masquer l'implantation de start-upsnationales du green business.

Pourtant, au-delà des chiffres officiels, on peut s'interroger sur le succès de la stratégie d'upgradingindustriel. La plupart des entreprises du secteur TIC qui arrivent installent des usines d'assemblage ou de fabrication. Les bureaux de R\&D restent à Pékin ou dans les villes côtières. Elles profitent d'un effet d'aubaines. Le salaire minimum est $50 \%$ moins élevé qu'à Shenzhen, le foncier est subventionné (les aides peuvent s'élever à $50 \%$ du prix du marché qui est déjà bien inférieur à ceux des villes côtières) et la main d'œuvre plus facile à trouver : le Sichuan est proche, et aujourd'hui, les mingongs ${ }^{16}$ sont plus réticentsà allerdans l'Est, loin de leurs familles.De plus les investissements publics ont été massifs : l'énergie et le coût du transport sont bon marché,l'environnement et le cadre de vie connaissent une amélioration. La Municipalité met en avant la possibilité pour la classe moyenne d'acquérir un appartement ensix ans et demi.

Mais onobserve le même processus de relocalisation des entreprises du secteur TIC vers la capitale du Sichuan,Chengdu, autre élément clé de la politique de Go West orchestrée par Pékin. Ainsi, Foxconn a ouvert en 2010 une immense usine de 120.000 travailleurs à Chengdu pour fabriquer l'Ipad d'Apple. On peut en conclure que la politique volontariste du gouvernement central rencontre les intérêts des industriels.

La question de la main d'œuvre qualifiée (manque d'experts et fuite des cerveaux) est un des principaux verrous relevés par notre enquête. Cette pénurie n'est pas propre à Chongqing et est quasi générale en Chine [Mercier-Suissa, Ruffier 2012] ; le système universitaire chinois et plus largement de formation a besoin de fournir un gros effort d'adaptation. Le plan gouvernemental sur le développement scientifique et technologique veut faire du pays une puissance technologique d'ici $2020: 4,3$ chercheurs pour 1000actifs, 2 millions de brevets par an, campagne de recrutement de 10.000 talents de recherche, construction d'un système national d'innovation pour donner la priorité aux entreprises comme moteur de l'innovation.

Mais d'autres facteurs de blocage apparaissent, à la frontière de l'économique et de la gouvernance.En Mars 2011, la municipalité de Chongqing, dans le but de développer le secteur high-tech, a annoncé son intention de construire une zone dédiée au cloud computing. Elle subventionne les implantations et garantie une totale liberté d'accès à Internet sur la zone, donc la possibilité de contourner leGreat Firewall [Puel, 2009]. La société singapourienne PacNet est choisie pour construire et gérer les infrastructures. Alors que le modèle du Guandong associe depuis plus de trente ans liberté économique et contrôle social avec des résultats remarquables, le modèle de Chongqing semble changer un peu l'équation. Gouvernement central et Municipalité semblent essayer de concilier des priorités potentiellement contradictoires : un rôle plus affirmé dans la régulation de l'économie et dans le même temps la promotion d'industries qui ne peuvent prospérer qu'avec une plus grande liberté de l'information et qui jusqu'alors ${ }^{17}$ se sont installés en Inde, Malaisie ou Singapour.Les racines de cet interventionnisme politique sont à la fois liées à l'histoire et

\footnotetext{
${ }^{16}$ Travailleurs migrants venus des campagnes sans hukou urbain (passeport ouvrant des droits).

${ }^{17}$ Seule IBM est installée àWuxi.
} 
contingentes aux récents aléas de la lutte politique : Chongqing était la base territoriale de Bo Xilai dans sa conquête avortée du pouvoir. Ce dernier exaltait le «retour aux sources » et faisait de l'intervention des autorités politiques dans la vie économique son thème favori.

La dimension sociale est difficile à évaluer en l'absence de données incontestables. Les autorités locales ou les chefs d'entreprises se félicitent de l'intégration de la paysannerie voire de l'augmentation du niveau de vie. Le salaire minimum a augmenté de 680 à 870 en 2011 puis à 1050 yuans en 2012. Mais on peut lire les faits autrement : expropriation forcée des paysans (indemnisation faible ou inexistante sur fond de violence), absence d'état de droit avec tout son cortège d'abus, corruption, une population flottante estimée à 4 ou 5 millions de personnes qui vit dans une grande précarité, etc.Ainsi,Chongqing apparaît dans la longue liste des émeutes urbaines. La dernière, en 2012, a conduit dans la rue des ouvriers et leurs familles victimes d'une dévalorisation de leurs terres suite à un réaménagement urbain et à la délocalisation d'un site de Hong-Yan Auto. Le développement de Chongqing n'échappe pas aux grandes tendances observées dans les métropoles chinoises : disparition accélérée de la mixité urbaine et fragmentation socio-spatiale généralisée [Wu et alii,2010; Puel et Fernandez 2012].

\section{Conclusion}

Face aux pressions internationales et de son opinion publique choquée par divers scandales environnementaux, l'État a engagé la transition vers un mode de développement plus sobre. Ce processus d'adaptation qui a débuté dans le domaine sensible de l'énergie, s'étend aujourd'hui à d'autres secteurs comme les TIC et concerne étroitement les modèles de développement des grandes métropoles. La Chine cherche un nouveau modèle d'industrialisation, à la fois plus sobre mais conciliant ses objectifs de développement économique et de rééquilibrage spatial ( $g o$ west) et social (intégration des populations rurales et développement de son marché intérieur). À Chongqing, ce modèle est mis en œuvre sous l'étroit contrôle de Pékin.

Ce volontarisme actif,inflexion du modèle du Guandong,explique en partie le rapide succès «quantitatif» mais les méthodes laissent sceptique si l'on aborde les autres aspects du développement durable,car le bilan provisoire montre beaucoup d'économie, peu d'environnement et encore moins de social.

Derrière les chiffres, se cache la politique des transferts territoriaux d'externalités négatives menée dans toutes les provinces chinoises de Pékin au Guandong. À l'échelle du pays, ils déplacent les activités vers l'Ouest, à l'échelle des régions, vers les territoires ruraux connexes dont les élites aspirent au développement, même au prix du sacrifice de leur environnement.

Les tentatives d'upgrading et de verdissement du secteur des TIC illustre toutes les contradictions. Comment satisfaire les marchés extérieurs et le développement du marché local dont on fait une priorité nationale en verdissant en enchérissant les coûts de production des TIC dans un contexte de concurrence mondiale et sans faire respecter un état de droit ?

Les progrès réalisés le sont souvent en raison de l'induction des règlementations des pays d'exportation ou au transfert de ces contraintes vers des pays sous-traitants. La mise en œuvre des lois et normes reste hasardeuse, mine la confiance dans le système global et freine l'implantation des activités les plus stratégiques. De plus, les systèmes de formation et de recherche sont en retard par rapport aux. Aussi il ne faut pas s'étonner que les relocalisations à Chongqing concernent principalement les activités de fabrication d'entreprises à la recherche de coûts optimisés et de la proximité du marché intérieur.

Notre étude souffre de trois limites majeures. Premièrement, limiter l'étude à l'exemple du secteur TIC, même si les autorités en ont fait un axe clé, introduit nécessairement un biais. Il serait intéressant d'analyserun autre secteur clé du territoire, comme l'automobile, dont le 
développement est également axé vers l'international et le marché intérieur et prétendument sur le verdissement de ses activités.Deuxièmement, le manque de recul ne permet pas de porter un jugement tranché sur la nature et le succès de ce modèle de développement ; l'analyse des premiers résultats nous permet simplement d'émettre des doutes sur la réalité de l'upgrading dans le secteur des TIC et sur l'effet levier du développement durable dont peu ou prou toutes les régions se réclament depuis 2010.

Enfin, pour les mêmes raisons, nous pouvons douter de la transférabilité d'un tel modèle à d'autres régions qui, vu le gigantisme des ambitions de production affichées, ne peut être que difficilement compatible aujourd'hui avec les objectifs du développement durable.

\section{Remerciements}

Le projet a été financé par l'ADEME (Agence de l'Environnement et de la Maîtrise de l'Énergie) et le MAE (bourse Eiffel du Ministère des Affaires Étrangères).Une thèse en cotutelle a réuni les deux Universités de Toulouseet de Chongqing.

\section{Références}

BECKERMAN W. [1992], «Economic growth and the environment: whose groth? whose environment ?» World Development, ${ }^{\circ} 20$, pp. 481-496.

BERKHOUT F., HERTIN J. [2001], Impacts of Information and Communication Technologies on Environmental Sustainability: speculations and evidence, reportto the OECD, 22p.

BIHOUIX P.,de GUILLEBON B. [2010], Quel futur pour les métaux? Raréfaction des métaux : un nouveau défi pour la société, EDP Sciences, 299 p.

BOUTAUD A., BRODHAG C., GONDRAN N. [2006],«Lorsque le développement perd le Nord ! Courbes de Kuznets Environnementales : l'apport des indicateurs alternatifs de type empreinte écologique dans la réflexion sur le développement durable », http: //ensieg.dox.free.fr/3A_SEM_OSI/D\%E9veloppement\%20Durable/Documents/ECKBoutaudBrodhag-2006.pdf, (consulté le 25/01/2013).

BREUIL H., BURETTE D., FLÜRY-HÉRARD B., CUEUGNIET J., VIGNOLLES D. [2008], Rapport TIC et développement durable, CGEDD (Conseil Général de l'Environnement et du Développement Durable) et CGTI (Conseil Général des Technologies de l'Information), $96 \mathrm{p}$.

CAO C. [2004], «L'industrie chinoise face au défi technologique »,Perspectives chinoises n83; mis en ligne le 01 mai 2007, http: //perspectiveschinoises.revues.org/1372, (consulté le 23 janvier 2013).

ENERTECH, [2008], Mesure de la consommation des usages domestiques de l'audiovisuel et de l'informatique, rapport du projet REMODECE, ADEME-Union européenne-EDF, $80 \mathrm{p}$.

FLIPO F., DELTOUR F., DOBRÉ M., MICHOT M. [2012], Peut-on croire aux TIC vertes ? Technologies numériques et crise environnementale, Presses des Mines, Paris, 272 p.

GADGIL M., GUHA R. [1995], Ecology and equity: The Use and Abuse of Nature in Contemporary India, Routledge, $213 \mathrm{p}$.

GARTNER., REYNOLDS M. [2008], Scalability and sustainability, OAGITM Conference, Fall, http://www.oagitm.org/conferences/08_fall_conference/MartinReynolds_OAGIT\%20Pr esentation.pdf, (consulté le 25/01/2013).

HAGELÜKEN C. [2008], « Mining our computers - opportunities \& challenges to recover scarce and valuable metals from electronic devices »,Electronic goes green 2008+ symposium, Berlin.

HUANG D. [2013], Economic analysis of ICT industry's eco-design and energy efficiency design in China with case of Chongqing City (à paraître), Doctorat de géographie- 
aménagement, Université de Toulouse, copie accessible auprès de Gilles Puel, UFR Sciences Space and Society, University of Toulouse II.

HUANG D.Y., LIU C., DRAETTA L., PUEL G. [2012], « La genèse de l'éco-conception en Chine dans le secteur des TIC, une exportation européenne »,VertigO - la revue électronique en sciences de l'environnement, Volume 12 Numéro 2 |URL: http: //vertigo.revues.org/12624 ; DOI : 10.4000/vertigo.12624, (consulté le 11 octobre 2012).

JOHNSON J., HARPER EM., LIFSET R., GRAEDEL TE. [2007], « Dining at the periodic table: metals concentrations as they relate to recycling », Environment Science \& Technology, 2007 Mar 1; 41(5): 1759-65.

KUZNETS S. [1955], «Economic Growth and Income Inequality »,The American Economic Review, vol. $45, \mathrm{n}^{\mathrm{o}} 1$, p. 1-28.

LACOUR P., SIMON J.C. [2012], «Quelle intégration des pays en développement dans le régime climatique ? Le Mécanisme pour un Développement Propre en Asie »,Développement Durable et territoires, 3 (2012) pp. 1-19.

LI Y., SHEPHERD J., LAYKE J., CURRIE J.[2012], Essential Buildings :The Emergence of "Low-Carbon Cities" in Post-Industrial Urban China, Institute for Building Efficiency,33 p. MARTINEZ-ALIER J.[1993], «Distributional Obstacles to International Environmental Policy: the Failures at Rio and Prospects after Rio »,Environmental Values, 2, p. 97-124.

MARTINEZ-ALIER J. [2002], The Environmentalism of the Poor, Cheltenham UK Northampton, MA, USA, Edward Elgar, 59 p.

MERCIER-SUISSA C., RUFFIER J. [2011], «Une politique "développement durable" peutelle favoriser le développement industriel ?: le cas du Guangdong ", in colloque Développement durable, Territoires et Localisation des entreprises: Vers une attractivité durable ?, http://halshs.archives-ouvertes.fr/halshs-00695508 (consulté le 25/01/2013).

NIJKAMP P., LASSCHUIT P., SOETEMAN F.[1992], "Sustainable Development in a Regional System »,inBREHENY M.J. [Dir.], Sustainable Development and Urban Form, Series, editor P.W.J. Batey, p. 39-66.

OLSZAK E. [2012], "Localisation des activités et développement durable des territoires : quelle interactivité», in CARDEBAT J.M., UZUNIDIS D. [Dir.], «Territoire vert : Entreprises, institutions, innovations », Marché et organisations n ${ }^{\circ} 16,2012 / 2$, pp 153-180.

PEARCE D., MARKANDYA A., BARBIER E.B.[1989], Blueprint for a Green Economy, éditionde 1992, London : Earthscan Publication Limited.

PUEL G. [2009], «Les politiques publiques de régulation de l'accès à Internet en Chine : conditions d'accès et modèles d'usages dans les cybercafés des villes chinoises », in CHÉNEAU-LOQUAY A. (Dir.), «Accès aux nouvelles technologies en Afrique et en Asie, TIC et service universel »,Les cahiers de Netsudsn ${ }^{\circ}$ août 2009, pp. 123-136.

PUEL G., FERNANDEZ V. [2012], «Socio-technical systems, public space and urban fragmentation: the case of "cybercafes" in China », in Urban studies, May 2012 Issue, Volume 49 (6). pp.1-17

SHUE V. [1988], The Reach of the State: Sketches of the Chinese Body Politic, Stanford, University Press, $175 \mathrm{p}$.

SUN L. [2012], L'analyse des téléphones Shanzhai, PUEL G. [Dir.], Master 2 en géographie des technologies, Université de Toulouse, copie accessible auprès de Gilles Puel, UFR Sciences Space and Society, University of Toulouse II, 46p.

WALLENBORN G., DOZZI J. [2007], « Du point de vue environnemental, ne vaut-il pas mieux être pauvre et mal informé que riche et conscientisé ? » in CORNUT P., BAULER T., ZACCAI E. (Dir.), «Environnement et inégalités sociales », Edition de l'Université de Bruxelles.

WU F., WEBSTER C., HE S., LIU Y. [2010],Urban Poverty in China, Edward Elgar Publishing, 240 p. 
ZHIYUE B., GANG C. [2009], « Bo Xilai and the Chongqing Model »,East Asian Policy, 4249, http://www.eai.nus.edu.sg/Vol1No3_BoZhiyueChenGang.pdf, consulté le 25/01/2013.

ZUINDEAU B. [2005], « Équité territoriale: quelles lectures par les théories du développement durable? »,Reflets et perspectives de la vie économique, 2005/4 Tome XLIV, p. $5-18$. 